\title{
Time-Restricted Feeding and Potential for Type 2 Diabetes Mellitus: A Narrative Review
}

Elijah Lustig, OMS II; Jay H. Shubrook, DO; Kim M. Pfotenhauer, DO

From Touro University College of Osteopathic Medicine, Vallejo, California (Student Doctor Lustig and Dr Shubrook) and Michigan State University College of Osteopathic Medicine, East Lansing, Michigan (Dr Pfotenhauer).

Disclaimer: Dr Shubrook, a JAOA associate editor, was not involved in the editorial review or decision to publish this article.

Financial Disclosures: None reported.

Support: None reported.

Address correspondence to

Elijah Lustig, OMS II, 1314 Court St, Alameda, CA $94501-4725$ Email:

elustig1995@gmail.com Submitted July 31,2019 ; revision received March 7, 2020; accepted April 6, 2020.
Intermittent fasting (IF) is an increasingly popular strategy for weight loss and improved metabolic health. IF regimes involve deliberate implementation of fasting windows ranging from 12 to 72 hours. During these fasting windows, individuals reduce their caloric intake by $75 \%$ to $100 \%$. Such strategies show promise and are increasingly socially popular, but many effects are not completely understood. Specifically, the effects of time-restricted feeding (TRF), a specific form of IF in which participants undergo daily fasting periods lasting about 16 hours, is among the most popular yet understudied regimens. This review finds that TRF may produce mild weight loss and improved function of insulin. TRF also likely produces cardiovascular effects, such as decreased blood pressure and fasting blood triglycerides. TRF, like other forms of IF, may encourage fat loss specifically when combined with exercise. The manuscripts identified in this narrative literature review were analyzed for the potential benefit for patients with type 2 diabetes mellitus, and limitations of summarized content were noted. While these studies support some suspected benefits of TRF, they also legitimize the need for future TRF studies, since conclusions are greatly limited by a paucity of evidence.

J Am Osteopath Assoc. 2020;120(9):560-567. Published online August 7, 2020.

doi:10.7556/jaoa.2020.101

Keywords: insulin resistance, intermittent fasting, obesity, T2DM

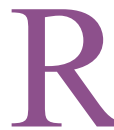
ates of obesity and associated metabolic diseases, including type 2 diabetes mellitus (T2DM), are at an all-time high. ${ }^{1}$ Although malnutrition was a major concern in the 19th century, obesity is becoming increasingly common in the 21 st century. $^{2}$ A 2015 study $^{3}$ used a smartphone application to monitor eating patterns, finding that most participants ate more erratically than self-predicted and that most had eating windows over 15 hours. These data suggest that overeating is on the rise and that current strategies for nutritional self-control and self-awareness are generally failing because they are not robust enough to prevent poor health outcomes.

Current clinical therapies for obesity and T2DM include lifestyle changes, medications and, in some cases, metabolic surgery. ${ }^{4-7}$ However, the benefits of medical and surgical treatments are accompanied by associated risks. Even lifestyle interventions seem hard to implement; many existing lifestyle therapies require constant, strict supervision of energy consumption and expenditure, often with much involvement of health care professionals. ${ }^{8}$ Medications have adverse effects and most come with a substantial financial 


\section{Table. \\ Examples of Intermittent Fasting Regimens ${ }^{15-17}$}

\begin{tabular}{ll} 
Regimen $^{\mathrm{a}}$ & Description \\
\hline Alternate day $^{\mathrm{b}}$ & Alternate between fasting days and normal eating days \\
\hline 5-day/2-day & 5 days of normal eating followed by 2 days of fasting \\
\hline Fast-mimicking diet & Low-protein, low-sugar diet administered for 5 days, once per month for 3 months \\
\hline $16 / 8$ time-restricted feeding & 16 hours of fasting, 8 hours of unrestricted eating \\
\hline
\end{tabular}

a Intermittent fasting strategies all allow consumption of water while fasting and typically black coffee and unsweetened tea.

b Fasting days in alternate-day studies ranged from $0 \%$ to $25 \%$ of normal calorie intake.

burden. ${ }^{9}$ Surgeries are invasive, carry immediate and long-term risks, and present tremendous cost. ${ }^{7}$ These drawbacks contribute to decreased adherence to lifestyle therapies, inevitably worsening patient outcomes. $^{10}$

Insulin resistance (IR) is a core pathophysiologic defect of T2DM and metabolic syndrome. ${ }^{1-14}$ However, much of contemporary T2DM management is focused on managing hyperglycemia, a symptom of the underlying IR. Therefore, an adherable, lifestylebased intervention that targets IR would provide potential value for management.

Many forms of intermittent fasting (IF) have been studied. ${ }^{15}$ Fasting programs have included alternate-day fasting, a 5-day/2-day diet, a fast-mimicking diet, and a 16-hour/8-hour time-restricted feeding (TRF) regimen. ${ }^{15-17}$ These fasting regimens are further explained in Table. The results vary, but forms of IF have shown benefits in mouse and human models. ${ }^{16,18-20}$ Cardiovascular risk-factor benefits include decreases in blood pressure, low-density lipoprotein (LDL) cholesterol, and total cholesterol when combined with caloric restriction. ${ }^{17,21}$ Moderate beneficial decreases to body weight and caloric intake seem to be generally on par with other methods of caloric restriction, but may preferentially encourage fat loss while maintaining lean mass ${ }^{16,18,22}$ and are more easily achievable for patients. ${ }^{17,19}$ Many of these effects would prove useful in the management of T2DM, especially when considering that many patients with T2DM have cardiovascular-related illness and are overweight or obese.

Metabolic changes that affect IR are likely a primary benefit of IF and may underlie many of the reported health improvements associated with IF. $8,18,23,24$ Studies of chrononutrition suggest a likely mechanism for many of these benefits. ${ }^{3,14}$ The established interplay between metabolism and circadian rhythm is greatly influenced by timing of nutritional intake. ${ }^{14,19}$ As such, it is likely that the metabolic effects of TRF, which increases proportions of daytime eating, arise from synchronizing nutritional intake with internal metabolic clock genes. ${ }^{14}$ Some of this increased alignment between circadian and metabolic pathways, specifically in the case of TRF, likely arises from reduced nighttime eating on many days of the week. ${ }^{3,14}$ If lack of synchronization can lead to metabolic syndrome and obesity, then IF regimens, specifically TRF, likely provide health benefits beyond those of caloric restriction. $^{19}$

TRF is a form of IF in which individuals limit daily eating windows to 8 hours \pm 1 hour, typically eating in windows beginning at $8 \mathrm{AM}$ or $12 \mathrm{PM}$, in part to limit nighttime caloric intake. Compared with traditional IF regimens, TRF uses shorter-duration fasting windows ( $16 \mathrm{~h} \pm 1 \mathrm{~h}$ ), but on a more frequent basis. The extent of benefits provided by TRF is still undetermined. Truly understanding the effects and possible uses of TRF holds promise not just for patients with obesity and T2DM, but for the medical community as a whole. 
This review aims to comprehensively summarize published scientific literature on the subject of TRF in humans. Particular attention is given to health improvements most pertinent to T2DM and insulin resistance. The results shown in this review can help busy health care professionals give evidence-based advice on this nutritional technique.

\section{Methods}

A literature search was conducted on June 28, 2019, using PubMed's advanced search. Reference citations in articles were also reviewed. For inclusion in this review, articles must have described original research using human participants and have been published in English. TRF was defined as intermittent fasting regimens in which experimental eating windows were 8 hour \pm 1 hour, with daily water-only fasts for a minimum of 1 week. The search terms "time-restricted feeding" and "time-restricted eating" were used.

\section{Results}

This PubMed search produced 129 articles. Of the 129 possible articles, 83 were excluded because they used animal models/subjects. Another 29 articles were excluded because they were reviews or summaries of previously published literature. Although unoriginal, these studies were read for context. Seven studies were disqualified because they did not study TRF as we defined it and instead examined other aspects of fasting or nutrition. Two articles (Gabel et $\mathrm{al}^{25}$ and Gabel et $\mathrm{al}^{26}$ ) did not meet all inclusion criteria but were highly applicable studies of TRF and, as such, were noted for relevance. The remaining 8 articles contained 2 repeated articles, resulting in 6 usable articles, 1 of which (as mentioned above) studied sleep quality during $\mathrm{TRF}^{25}$ and is not discussed.

\section{Summary of Reviewed Studies}

Our search produced 6 articles that described 4 unique studies. The studies tended to investigate TRF as it pertained to areas of nutrition and metabolic and cardiovascular disease. Additionally, some articles investigated potential implications for inflammatory and rheumatoid disease. Many disqualified articles still provided relevant information that supported or validated conclusions drawn by the qualifying articles. The most important variables investigated in the qualifying studies included body weight, body mass index, blood pressure; fasting blood glucose, fasting blood insulin, fasting blood triglycerides, glucose, and insulin levels; and triglyceride
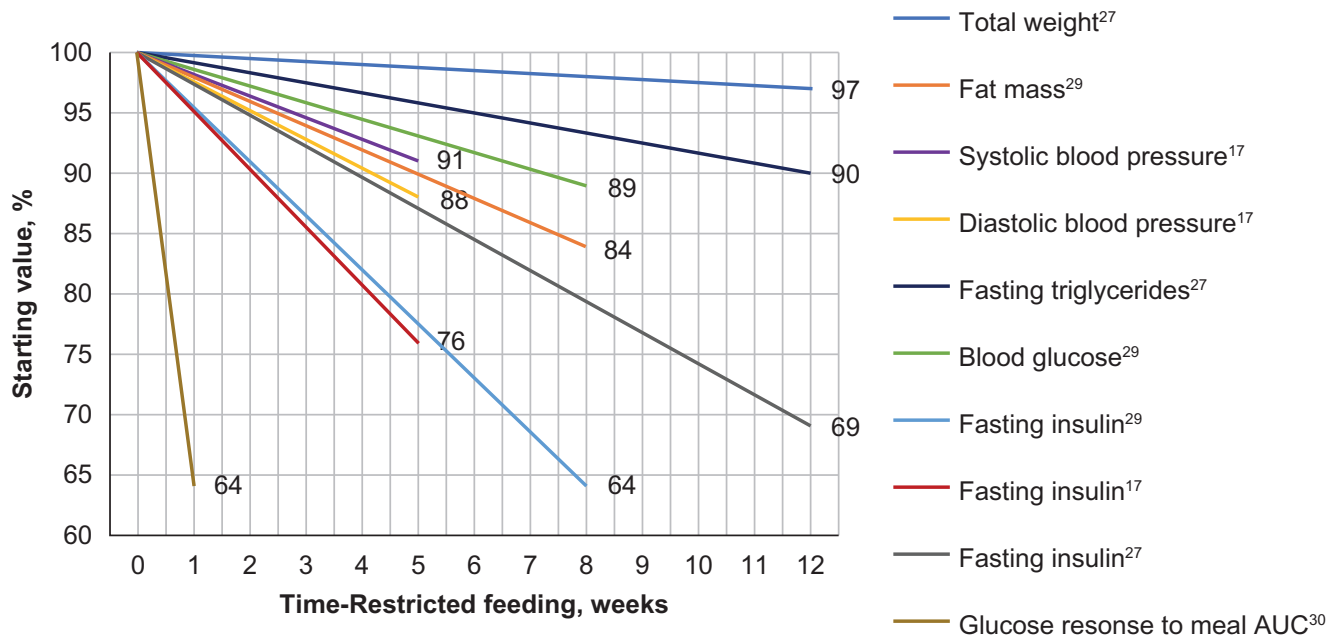

Figure.

Metabolic changes seen in time-restricted eating studies. Values are shown as percentage of change over time. ${ }^{17,26,28,29}$ 
responses to meals. The results for these variables are summarized in the Figure, which provides a visual summary of the potential benefits most pertinent to T2DM. As seen in the Figure, TRF has the potential for improving several key markers of health. One of the most consistent results reported is a decrease in fasting insulin level, which supports the theory that TRF may improve insulin function.

\section{Gabel et $\mathbf{a l}^{25,26}$}

This study produced 3 published articles. ${ }^{25-27}$ Twenty-three participants who were obese (20 women, 3 men) participated in a TRF regimen (ad libitum eating from $10 \mathrm{AM}$ to $6 \mathrm{PM}$ and water-only fast from $6 \mathrm{PM}$ to $10 \mathrm{AM}$ ) every day for 12 weeks. ${ }^{26}$ Outcomes were compared with a matched historical control group. TRF participants lost weight (mean [SD], $-2.6 \%[0.5] ; P<.001)$, had reduced energy intake (mean [SD], $-341 \mathrm{kcal} / \mathrm{d}$ [53]; $P=.04$ ), and had decreases in systolic blood pressure (mean [SD], 7 [2] $\mathrm{mm} \mathrm{Hg} ; P=.02$ ). These findings suggest that TRF produces a mild caloric deficit and weight loss without calorie counting and may lower blood pressure. An analysis of the safety of the experiment was also published. No serious adverse events occurred in any participant, supporting the belief that TRF is likely safe.

\section{Hutchison et $\mathbf{a l}^{28}$}

Fifteen men at risk for T2DM underwent 2 different 7-day TRF regimens, either an early TRF schedule (caloric intake confined to $8 \mathrm{AM}$ to $5 \mathrm{PM}$ ) or a delayed TRF (caloric intake confined to $12 \mathrm{PM}$ to $9 \mathrm{PM}$ ). ${ }^{28}$ Outcomes were compared with baseline after a 2 -week washout period. TRF reduced glucose response to meals by $36 \%$ (mean [SEM], -1.6 [.4] mmol/L; $P=.001$ ) as measured by incremental area under the curve (iAUC) of plots generated from venipuncture samples. TRF also demonstrated a trend to decrease insulin iniAUC $(P=.09)$. TRF decreased fasting triglycerides from a mean (SEM) of $1.3(0.1) \mathrm{mmol} / 1$ to 1.1 $(0.1) \mathrm{mmol} / \mathrm{l}$ in early TRF, and from $1.2(0.2) \mathrm{mmol} / \mathrm{l}$ to $1.1(0.1) \mathrm{mmol} / 1$ in delayed TRF $(P=.003)$ but not by triglyceride iAUC in response to a meal $(P=.57)$. Participants reported adhering to the diet about 6 days per week. Overall, TRF improved glycemic responses in individuals. Additionally, their results manifested without significant decrease in body weight, suggesting benefits of TRF may exist independently of weight loss.

\section{Moro et $\mathbf{a l}^{29}$}

Thirty-four resistance-trained men were randomly assigned TRF (caloric intake confined from 1 PM to 8 PM) or normal diet (caloric intake confined to 8 AM to 8 PM). ${ }^{29}$ Groups were matched for energy intake and macronutrient distribution. Participant weight was actively maintained, meaning that experimenters adjusted caloric intake over time to minimize any weight gain or loss. TRF caused a mean (SD) of 0.96 (1.76) $\mathrm{kg}(P=.007)$ decrease in fat mass, although fat-free mass, muscle area, and maximal strength did not significantly change. Testosterone (mean [SD], -2.07 [3.6] nmol/L; $P<.0001$ ) and IGF-1 (mean [SD], -12.38 [33.0] ng/L; $P=.0003$ ) decreased significantly in patients following the TRF. TRF, when combined with resistance training, significantly reduced fat mass while maintaining muscle mass. Participants in this study were healthy, resistance-trained men and, therefore, differed from the typical T2DM population. This study particularly elucidates some of TRF's potential to encourage healthy body composition, especially when combined with physical activity. ${ }^{17}$

\section{Sutton et al $^{17}$}

Eight men with prediabetes who were considered overweight underwent 5 weeks of TRF (water-only fast from $8 \mathrm{AM}$ to $3 \mathrm{PM}$ ) compared with a normal diet (12hr eating window, beginning 6:30 AM to 8:30 AM ) in a randomized order, separated by a 7-week washout period. ${ }^{17}$ Participants were fed a diet that was isocaloric and eucaloric to prevent changes in body weight. Researchers deliberately maintained participants' weight to investigate effects of fasting independent of weight loss. TRF elicited significant decreases in mean insulin and IR while also improving B-cell 
responsiveness. TRF also caused a notable decrease in mean (SD) systolic and diastolic blood pressure of 11/ 10 (4.0) $\mathrm{mm} \mathrm{Hg}(P=.03)$. This study precipitated no serious adverse events. All but 1 participant reported that TRF was not difficult or only moderately difficult. In a poststudy survey, participants responded that about 8 hours would be an achievable eating window. These results further support the notion that TRF provides various benefits beyond those associated with weight loss. Additionally, this trial confirms theories that TRF is safe and widely achievable.

\section{Discussion}

Although the evidence on TRF is rather limited, it does appear to present notable potential benefits. These results are consistent with expectations formed by results of similar IF studies. ${ }^{16,22}$ The primary effect of decreased IR is seemingly reliable and is replicated in other related studies. ${ }^{16,22}$ Considering that the TRF regimen is accessible to almost everybody, widely achievable, and carries little to no financial burden, any small benefit of TRF comes with little downside. Pertinent results from the studies reviewed here demonstrate safety and attainability, with beneficial cardiovascular, metabolic, and weight-related nutritional effects.

TRF, consistent with findings of studies on other forms of IF, seems to be safe and well tolerated. Studies reviewed here reported no serious adverse events and concluded that TRF is safe for most participants. ${ }^{17,27}$ These studies boasted high compliance totaling over $97 \%$, suggesting that participants had no trouble adhering to TRF at least 6 days per week. ${ }^{17,26}$ One study reported that TRF did not significantly increase evening hunger and that fasting for 16 hours was not as hard as individuals expected. ${ }^{17}$ These findings begin to elucidate why TRF is so achievable for most participants and might even shed light on why TRF and IF have begun to become prominent in popular culture.

TRF shows several possible cardiovascular risk factor benefits, including significantly lower blood pressure and likely lower fasting triglyceride levels. ${ }^{28,29}$ One study ${ }^{17}$ found that TRF increased triglyceride level, but these results were not matched to time; researchers of this study specifically mentioned that they believed the elevated triglyceride level to be transient and likely due to the increased fat mobilization thataccompanied weight loss. TRF has shown no effect to date on total cholesterol, HDL cholesterol, and LDL cholesterol levels. ${ }^{17,29}$

The metabolic effects of TRF may include improved insulin function and improved $\beta$-cell islet function as measured by insulinogenic index. ${ }^{16}$ TRF may reduce fasting glucose level; this result was found to some degree in 2 different studies. ${ }^{28,29}$ What is more certain is that TRF reduces IR, leading to improved insulin functioning. ${ }^{16,26,29}$ This finding is especially relevant because it indicates that TRF may serve as a treatment for IR, a core defect of T2DM, as opposed to correcting hyperglycemia, a symptom.

Much of the cultural and social excitement over IF revolves around its potential for reducing body weight while improving body composition. One study did reveal the potential for slow but steady weight loss: participants lost $3 \%$ of their body weight during 12 weeks. ${ }^{26}$ More important is the potential for TRF to preferentially encourage fat loss with maintenance of lean mass, especially when combined with resistance training. Moro et $\mathrm{al}^{29}$ found that 34 resistance-trained men who trained with resistance lost $16.4 \%$ of their body fat, even when total body weight was maintained. This finding conforms to a 2013 study that reported more fat loss and decreased lean mass in IF regimens compared with calorie restriction. ${ }^{19}$ When investigating the effects of TRF on daily caloric deficit, it appears that the deficit associated with a single day of breakfast omission is approximately maintained over weeks and months. $^{26,30,31}$ This implies that skipping breakfast does indeed produce a caloric deficit, and this deficit is maintained long term, leading to progressive weight loss. As mentioned, the majority of weight loss seemed to be of adipose tissue, especially when combined with resistance training. ${ }^{26,29}$ 
One of the most relevant nuances of the data reviewed here is that TRF seems to have benefits independent of weight lost during the regimen, because multiple studies reported benefits even when participant weight was actively maintained. This finding reveals that TRF helps participants not only because of what they eat, but also because of when they are eating. TRF has the potential to treat certain nutritional diseases and improve metabolic function through both temporal and quantitative caloric restriction.

\section{Limitations of the Research Findings}

IF is regarded by some as a popular diet, ${ }^{32}$ and has been part of religious and cultural practices for thousands of years. Although the popularity of TRF is growing, the body of research on this topic is still quite small. The literature that exists on the topic of fasting largely investigates cellular models or socially uncommon fasting regimens. TRF, among the most popular regimens in social concepts of IF, has been investigated by a limited number of studies. Additionally, because the research team limited the search to PubMed, it is possible that other applicable articles may have been listed in other databases.

The studies we reviewed confirmed some of the theorized benefits of TRF; however, they also revealed that these benefits may be only moderate in magnitude. Additionally, the dearth of widely generalizable evidence means that more studies must be conducted before physicians can be confident in understanding the risks and benefits of TRF. Future studies should investigate the effects on women, because most of the studies we reviewed results with men only. In addition, because pilot studies have turned out to be successful and safe, future studies should include more participants and longer periods of TRF. It would be of significant interest to further understand the safety and long-term effects of TRF; tracking of adverse events warrants future study, with long-term follow-up to analyze whether and how benefits are maintained. The literature, although present, is indeed sparse, and thus TRF should be used by patients with caution and careful observation by physicians. The extent of health benefits provided by TRF still needs to be fully explored. With these limitations in mind, it is important to note that TRF is seemingly safe in most adult populations and, as such, should not bediscouraged.

\section{Implications for Patients With T2DM}

TRF, as a long-term adjunct treatment, may be an effective, safe, and attainable method of reducing body weight, blood pressure, and IR in patients with T2DM. Although fasting-based interventions may not be suitable for all patients, research does suggest that fasting may provide various health benefits for patients diagnosed with, or hoping to prevent, T2DM and other chronic metabolic diseases. ${ }^{16,18,33}$ TRF holds potential for reducing IR, a core defect of T2DM that is often ignored or undertreated. Therefore, TRF proves a good place to start for T2DM patients who are interested in or open to fasting as a therapy. Patients will likely find the regimen relatively easy, contributing to achievability. ${ }^{17}$ Medications should be monitored closely by health care teams for opportunities to lower doses. This is essential for avoiding possible hypoglycemic episodes. Patients should continue to follow all medical direction and advice from their provider. Patients and health care professionals should closely monitor blood sugar. Patients with hypoglycemic unawareness may not be suitable for this treatment.

\section{Osteopathic Philosophy}

Benefits of IF can be seen as an example of the second osteopathic tenet, that the body is capable of selfregulation, self-healing, and health maintenance. Removing a potentially harmful block to natural health in this case, a lengthy eating window ${ }^{3}$ - can promote health by enabling the body's own mechanisms of healing and self-maintenance. ${ }^{28}$ By aligning behavioral eating windows with times of maximal metabolic capability, as influenced by circadian rhythms ${ }^{14}$ and microbiome, ${ }^{34,35}$ physicians may use the human body's own mechanisms of self-healing, and TRF may be an effective initial fasting therapy for certain patients. 


\section{Conclusion}

TRF shows promise as a treatment for those with T2DM and warrants further investigation. Although little research exists on the effects of TRF in patients with T2DM, current studies indicate it is somewhat effective, typically safe, and achievable. While not all effects of TRF are fully understood, improved insulin function, and steady weight loss are emerging as primary effects. There is also evidence to suggest TRF provides other metabolic and cardiovascular benefits. Additionally, TRF may be a management option that patients find easily adherable, and thus, it may be an effective patient-centered treatment. Patients attempting TRF must monitor blood glucose closely, as the risk of hypoglycemia is present, although seemingly minimal.

\section{Author Contributions}

All authors provided substantial contributions to conception and design, acquisition of data, or analysis and interpretation of data; all authors drafted the article or revised it critically for important intellectual content; all authors gave final approval of the version of the article to be published; and all authors agree to be accountable for all aspects of the work in ensuring that questions related to the accuracy or integrity of any part of the work are appropriately investigated and resolved.

\section{References}

1. Adult obesity facts. Centers for Disease Control and Prevention website; 2018. https://www.cdc.gov/obesity/data/adult.html. Accessed October 22, 2019

2. Hales CM, Carroll MD, Fryar CD, Ogden CL. Prevalence of obesity among adults and youth: United States, 2015-2016. NCHS data brief, no 288. National Center for Health Statistics; 2017. Accessed August 5, 2020. https://www.cdc.gov/nchs/data/databriefs/ db288.pdf

3. Gill S, Panda S. A smartphone app reveals erratic diurnal eating patterns in humans that can be modulated for health benefits. Cell Metab. 2015;22(5):789-798. doi:10.1016/j.cmet.2015.09.005

4. DeFronzo RA. Pharmacologic therapy for type 2 diabetes mellitus. Ann Intern Med. 1999;131(4):281-303. doi:10.7326/ 0003-4819-131-4-199908170-00008

5. DeFronzo RA, Eldor R, Abdul-Ghani M. Pathophysiologic approach to therapy in patients with newly diagnosed type 2 diabetes. Diabetes Care. 2013;36 (suppl 2):S127-138. doi:10.2337/dcS13-2011

6. Campbell RK. Type 2 diabetes: where we are today: an overview of disease burden, current treatments, and treatment strategies. J Am Pharm Assoc (2003). 2009;49 (suppl 1):S3-9. doi:10.1331/ JAPhA.2009.09077

7. Dixon JB, Zimmet P, Alberti KG, Rubino F, International Diabetes Federation Taskforce on E, Prevention. Bariatric surgery: an IDF statement for obese type 2 diabetes. Diabet Med. 2011;28(6):628-642. doi:10.1111/j.1464-5491.2011.03306.x

8. Chaix A, Zarrinpar A, Miu P, Panda S. Time-restricted feeding is a preventative and therapeutic intervention against diverse nutritional challenges. Cell Metab. 2014;20(6):991-1005. doi:10.1016/j. cmet.2014.11.001

9. Carver $\mathrm{C}$. Insulin treatment and the problem of weight gain in type 2 diabetes. Diabetes Educ. 2006;32(6):910-917. doi:10.1177/ 0145721706294259

10. Moreau A, Aroles V, Souweine G, et al. Patient versus general practitioner perception of problems with treatment adherence in type 2 diabetes: from adherence to concordance. Eur J Gen Pract. 2009;15 (3):147-153. doi:10.3109/13814780903329510

11. DeFronzo RA, Ferrannini E, Groop L, et al. Type 2 diabetes mellitus Nat Rev Dis Primers. 2015;1:15019. 2015;1:15019. doi:10.1038/ nrdp.2015.19

12. Kahn SE. The relative contributions of insulin resistance and beta-cel dysfunction to the pathophysiology of type 2 diabetes. Diabetologia. 2003;46(1):3-19. doi:10.1007/s00125-002-1009-0

13. Goldstein BJ. Insulin resistance as the core defect in type 2 diabetes mellitus. Am J Cardiol. 2002;90(5A):3G-10G. doi:10.1016/s0002-9149 (02)02553-5

14. Oike $\mathrm{H}$, Oishi K, Kobori M. Nutrients, clock genes, and chrononutrition Curr Nutr Rep. 2014;3:204-212. doi:10.1007/s13668-014-0082-6

15. Horne BD, Muhlestein JB, Anderson JL. Health effects of intermittent fasting: hormesis or harm: a systematic review. Am J Clin Nutr. 2015;102(2):464-470. doi:10.3945/ajcn.115.109553

16. Barnosky AR, Hoddy KK, Unterman TG, Varady KA. Intermittent fasting vs daily calorie restriction for type 2 diabetes prevention: a review of human findings. Trans/ Res. 2014;164(4):P302-P311. doi:10.1016/j.trsl.2014.05.013

17. Sutton EF, Beyl R, Early KS, Cefalu WT, Ravussin E, Peterson CM. Early time-restricted feeding improves insulin sensitivity, blood pressure, and oxidative stress even without weight loss in men with prediabetes. Cell Metab. 2018;27(6):1212-1221.e3. e1213. doi:10.1016/j.cmet.2018.04.010

18. Varady KA. Impact of intermittent fasting on glucose homeostasis Curr Opin Clin Nutr Metab Care. 2016;19(4):300-302. doi:10.1097/ MCO. 0000000000000291

19. Garaulet M, Gomez-Abellan P, Alburquerque-Bejar JJ, Lee YC Ordovás JM, Scheer FAJL. Timing of food intake predicts weight loss effectiveness. Int J Obes (Lond). 2013;37(4):604-611. doi:10.1038/ ijo.2012.229

20. Longo VD, Panda S. Fasting, circadian rhythms, and time-restricted feeding in healthy lifespan. Cell Metab. 2016;23(6):1048-1059. doi:10.1016/j.cmet.2016.06.001

21. Klempel MC, Kroeger CM, Bhutani S, Trepanowski JF, Varady KA. Intermittent fasting combined with calorie restriction is effective for weight loss and cardio-protection in obese women. Nutr J. 2012;11:98. doi:10.1186/1475-2891-11-98

22. Varady KA. Intermittent versus daily calorie restriction: which diet regimen is more effective for weight loss? Obes Rev. 2011;12(7): e593-e601. doi:10.1111/j.1467-789X.2011.00873.x

23. Longo VD, Mattson MP. Fasting: molecular mechanisms and clinical applications. Cell Metab. 2014;19(2):181-192. doi:10.1016/j. cmet.2013.12.008 
24. Rothschild J, Hoddy KK, Jambazian P, Varady KA. Time-restricted feeding and risk of metabolic disease: a review of human and animal studies. Nutr Rev. 2014;72(5):308-318. doi:10.1111/nure.12104

25. Gabel K, Hoddy K, Burgess HJ, Varady KA. Effect of 8-hour time-restricted feeding on sleep quality and duration in adults with obesity. Appl Physiol Nutr Metab. 2019;44(8):903-906. doi:10.1139/ apnm-2019-0032

26. Gabel K, Hoddy KK, Haggerty N, et al. Effects of 8-hour time restricted feeding on body weight and metabolic disease risk factors in obese adults: a pilot study. Nutr Healthy Aging. 2018;4(4):345-353. doi:10.3233/NHA-170036

27. Gabel K, Hoddy KK, Varady KA. Safety of 8-h time restricted feeding in adults with obesity. Appl Physiol Nutr Metab. 2019;44(1):107-109. doi:10.1139/apnm-2018-0389

28. Hutchison AT, Regmi P, Manoogian ENC, et al. Time-restricted feeding improves glucose tolerance in men at risk for type 2 diabetes: a randomized crossover trial. Obesity (Silver Spring). 2019;27 (5):724-732. doi:10.1002/oby.22449

29. Moro T, Tinsley G, Bianco A, et al. Effects of eight weeks of time-restricted feeding (16/8) on basal metabolism, maximal strength, body composition, inflammation, and cardiovascular risk factors in resistance-trained males. J Trans/ Med. 2016;14(1):290. doi:10.1186/ s12967-016-1044-0
30. Chowdhury EA, Richardson JD, Tsintzas K, Thompson D, Betts JA. Carbohydrate-rich breakfast attenuates glycaemic, insulinaemic and ghrelin response to ad libitum lunch relative to morning fasting in lean adults. Br J Nutr. 2015;114(1):98-107. doi:10.1017/S0007114515001506

31. Chowdhury EA, Richardson JD, Tsintzas K, Thompson D, Betts JA Effect of extended morning fasting upon ad libitum lunch intake and associated metabolic and hormonal responses in obese adults. Int J Obes (Lond). 2016;40(2):305-311. doi:10.1038/ijo.2015.154

32. Freire R. Scientific evidence of diets for weight loss: different macronutrient composition, intermittent fasting, and popular diets. Nutrition. 2020;69:110549. doi:10.1016/j.nut.2019.07.001

33. Chaix A, Manoogian ENC, Melkani GC, Panda S. Time-Restricted Eating to Prevent and Manage Chronic Metabolic Diseases. Annu Rev Nutr. 2019. doi:10.1146/annurev-nutr-082018-124320

34. Liu Z, Dai X, Zhang H, et al. Gut microbiota mediates intermittentfasting alleviation of diabetes-induced cognitive impairment. Nat Commun. 2020;11(1):855. doi:10.1038/s41467-020-14676-4

35. Kaczmarek JL, Thompson SV, Holscher HD. Complex interactions of circadian rhythms, eating behaviors, and the gastrointestinal microbiota and their potential impact on health. Nutr Rev. 2017;75 (9):673-682. doi:10.1093/nutrit/nux036

(c) 2020 American Osteopathic Association

\section{@TheJAOA on Twitter}

Follow us at http://www.twitter.com/TheJAOA to get involved and stay connected with updates, highlights, and conversations about osteopathic medical research. 